\title{
Perceptual and Computational Time Models in Game Design for Time Orientation in Learning Disabilities
}

\author{
Geert Langereis, Jun Hu, Pongpanote Gongsook, and Matthias Rauterberg \\ Department of Industrial Design, Eindhoven University of Technology \\ \{g.r.langereis, j.hu,p.gongsook, g.w.m.rauterberg\} @ tue.nl
}

\begin{abstract}
Several empirical studies confirm the importance of time-awareness in learning disabilities. Designed tools and environments to interact with time are essential for the training and diagnosis of an impaired notion of time. Training which improves sense of time may improve learning, daily functioning and quality of life. This paper reviews perceptual and computational time models in literature and present briefly our first attempts in applying the knowledge in the design of playful tools for children to orient their time.
\end{abstract}

Keywords: time models, time perception, learning disability, game design.

\section{Introduction}

Time perception is an abstract capability that facilitates the ability to predict, anticipate, and respond efficiently to coming events. For example, the preparation of fast responses benefit from the ability to predict precisely the point in time when an impending event requires a response. Also, precise representation of temporal information is required for the ability to organize and plan sequences of actions, particularly when sequences of novel or unskilled movements are required [1-3].

Several empirical studies confirm the importance of time orientation of children with learning disabilities [4]. The difficulty for processing time in its different dimensions has been shown to be an interesting universal characteristic of the dyslexic individual with reading problems. Furthermore, other conditions like Attention Deficit Disorders with hyperactivity (ADHD) appear to be characterized by comparable dysfunctions in time orientation.

Efforts in improving the time concepts of children in game design can already be found in many commercial or free products. Using a clock is a straightforward approach, such as the 24 Hour Analog Clock app for Android platforms [5]. TimeBuddy [6] from GoLearn International Inc tries to teach children about their daily routines and rhythms by using flash cards and stickers. Many interactive games are also available, for example Clockworks by BBC [7], teaching children how to tell time from a clock. On the website of free-training-tutorial.com, there are many similar "telling time" online games, most of which are using clocks as indicators of time or a time span [8]. Searching on Google with the keyword "time games for kids" returns a huge amount of hits, many of which are online games. A similar search using 
keywords "time games" and "learning disabilities" returns almost no links to products but concerns. A further investigation on these games discovers that most of these games are aims at teaching normal children using a straightforward clock concept without referring to or being based on clear and solid learning and time perception theories. Hardly any of them are designed for children with learning disabilities.

In interactive computer games for time orientation, instance and interval based computational computer models are applied in these games, either explicitly or implicitly. However while the goal of the games is to work on the mental models of the perceptual time, the computational models work at a lower level for the need of driving the games with time computation. Next we first review in the literature the concepts of the perceptual time models, followed by computational models. We then present three design cases where these time models are taken into account, as the first attempts in applying the knowledge in game design for children to orient their time to deal with learning disabilities.

\section{Perceptual Models}

Perceptual time models try to explain how time perception operates. In the literature, there are basically two categories of perceptual time models, depending on whether timing is seen as the output of a dedicated system. Grondin provides a good overview of these models in his literature review about timing and time perception [9], as summarized in Table 1.

Table 1. Perceptual time models [9]

\begin{tabular}{|l|l|l|}
\hline \multirow{2}{*}{$\begin{array}{l}\text { No } \\
\text { Central Clock }\end{array}$} & intrinsic models & $\begin{array}{l}\text { Timing is dependent specifically on a modality or a } \\
\text { coordination-dependent system }\end{array}$ \\
\cline { 2 - 3 } $\begin{array}{l}\text { With } \\
\text { Internal Clock }\end{array}$ & State-dependent network & $\begin{array}{l}\text { Timing does not depend on a clock, but on time- } \\
\text { dependent changes in the state of neural networks }\end{array}$ \\
\cline { 2 - 3 } & Pacemaker-counter models & $\begin{array}{l}\text { There are temporal intervals embedded in sequences } \\
\text { of signals. }\end{array}$ \\
\hline $\begin{array}{l}\text { The pacemaker emits pulses that are accumulated in } \\
\text { counter, and the number of pulses counted } \\
\text { determines the perceived length of an interval. }\end{array}$ \\
\hline
\end{tabular}

The purpose of time perception in human beings is to become adaptive in behavior, because it facilitates the ability to predict, anticipate, and respond efficiently to future events. The first thing children learn in primary school is what day it is, what month and what year. This is preceded by the notion of day and night. This all includes the ability of time orientation. At a shorter timescale, the ability of time estimation is needed. To prepare for a fast response we have to develop the ability to predict the point in time when an event requires a response. A third time perception aspect helps us to perform executive tasks and to have a social life. Precise representation of temporal information is required for the ability to organize and plan a series of actions, particularly when such a sequence exists out of new or fairly-new actions. These three aspects of time are summarized in Table 2. The three categories differ in how the brain has to process the concept of time and differ in the time span. 
Table 2. Methods to study timing and time

\begin{tabular}{|l|l|l|}
\hline & Perception aspect & Methods \\
\hline 1 & Time orientation (day, hour) & Zimbardo Time Perspective Inventory (ZTPI) \\
\hline 2 & $\begin{array}{l}\text { Time estimation (duration) or } \\
\text { retrospective timing }\end{array}$ & $\begin{array}{l}\text { Temporal order judgements related to auditory functions } \\
\text { and temporal tasks }\end{array}$ \\
\hline 3 & Planning or prospective timing & $\begin{array}{l}\text { Short intervals (sec): prospective timing } \\
\text { Long intervals: prospective memory and executive tasks }\end{array}$ \\
\hline
\end{tabular}

This overview provides a mapping for referencing to the different ways we interpret time perception. The orientation in time (absolute time) is described by the Zimbardo Time Perception Inventory (ZTPI) which provides an overview of how people remember and refer to time concepts [10].

Time estimation with respect to duration is a relative time concept. It involves the ability of retrospective timing and focuses on the estimation of past actions based on temporal judgement. This is based on short momentum in seconds or minutes [9].

Planning, or prospective timing, is orientated towards the future and can be discriminated in short and long term prospective timing. Short-term focuses on the timing itself while long-term prospective timing relates to memory [9].

Time perception has been assumed to be impaired in children with learning disabilities and some developmental impairment. Also, the occurrence of sleep disturbances in children with learning disabilities might be related to time concepts. For example, time orientation deficits have been studied with children with dyslexia, dyscalculia, autism, ADHD [4], and Duchenne Syndrome. However, the causal relation between training (the lack of) time perception and the impairment remains unclear.

\section{Computational Models}

Having looked into the perceptual models, we try to get an overview how timing and time are modeled in in multimedia systems, since most of the interactive games designed to improve children's time orientation are multi-modal by using multimedia content. $\mathrm{Hu}$ provides an extensive overview [11] based on the literature.

A multimedia system is characterized by integrated computer-controlled content generation, storage, communication, manipulation and presentation of independent time-dependent and time-independent media objects. Timing sits at the heart of multimedia systems, describing and managing the temporal relations between these media objects [12]. In multimedia systems, two representations of temporal relations can be indicated. These are based on instants and intervals. A time instant is a zerolength moment in time, such as "9:30am". By contrast, a time interval is defined by two time instants and therefore, their duration (e.g., "10s" or "9:30am to 9:40am").

Both instant and interval based models rely explicitly or implicitly on a timeline, which is often driven by a discrete computer clock. Comparing to the conceptual models in Table 1, one can easily conclude that these computational models work well with the perceptual models that do assume an internal clock. The perceptual models do not assume a central clock. They can therefore be implemented into state 
machine models or event driven models in computation. Although they are not necessarily described in the documentations of multimedia systems as basic timing mechanisms, these models are well supported by most of the computation platforms.

\section{Design Cases}

In the previous sections, we have seen there are indications of a link between time perception and some classes of learning and developmental disorders, and the perceptual time models are well supported by time models in multimedia systems. We have also seen that the concept of "time" plays a crucial role in computer architectures. This yields the assumption that there are design opportunities to create environments or products to interact with time. For example, in Virtual Reality environments, there are options to deviate from a linear progress of time [13]. We can switch in minutes from day to night or from summer to winter, or even reverse time. In addition, by using the multiple forms of product semantics, we may be able to interact with time by means of tangible products. In the following design cases, we show some examples of projects where products were designed with the aim to interact with time.

\subsection{Retrospective Timing}

The designed time interaction tool consists of a three-button input device and a software program (Fig. 1). The user has to memorize tone patterns of no more than three sounds that are supported by colored blocks on a screen. After a few seconds of pause, the sound has to be reproduced by pushing the buttons. The assumption is that children with dyslexia perform worse on the task, and show a slower learning curve for the accuracy of the task. An instant based time model is used to implement the program, but the intervals are derived from the time instants for the children to estimate the time span of the sound.

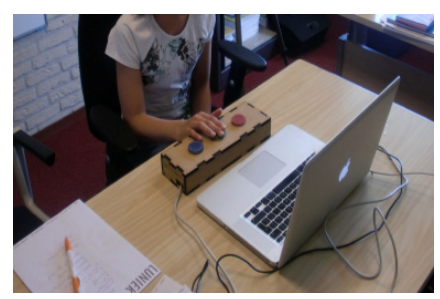

Fig. 1. Time interaction tool

\subsection{A Timer for Parent-Child Interaction}

When the parents tell their children to go to bed within five minutes, the concept of "five minutes" is hard to understand for a young child. A product is developed that visualizes time in an abstract way such that time becomes less tight and strict (Fig. 2). 
The goal of this product is to introduce more structure in the daily life of parents with their baby/child. Through this product, the child learns to understand and deal with the concept of time, especially the time intervals which are essential for planning.

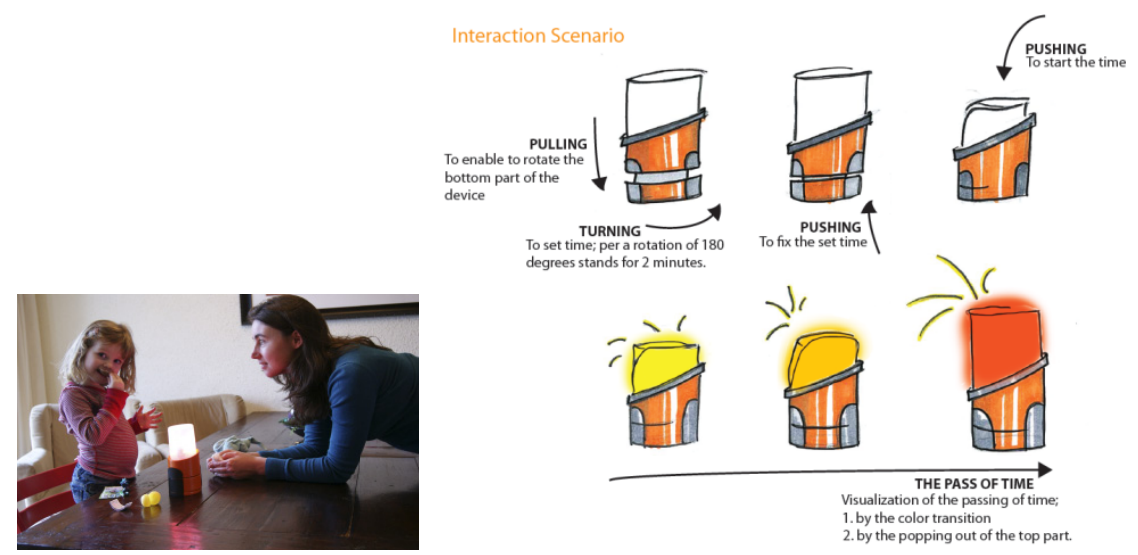

Fig. 2. A timer for parent-child interaction

\subsection{Management of ADHD in Classrooms}

In another project, we developed an interactive tool that helps children with Attention Deficit Hyperactivity Disorder (ADHD) to make fruitful use of breaks. Children with ADHD have concentration problems, but also problems with planning (so timing) of executive tasks. The interaction tool uses power breaks in a classroom to reflect and to plan the executive task in a playful way (Fig. 3). In this design, both time instants and intervals are important for the children to make proper planning.

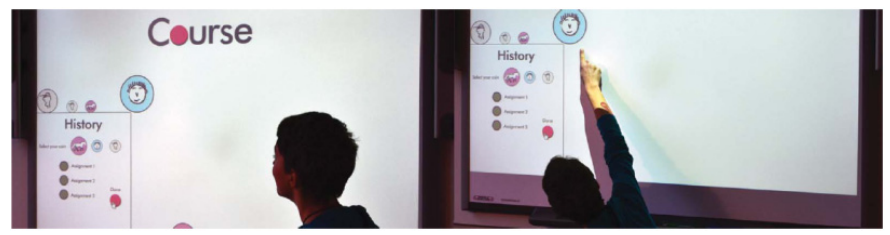

Fig. 3. Management of ADHD in classrooms

\section{Conclusions}

According to literature, there are basically two types of perceptual timing models in humans, with or without a central clock. We think all these timing models shall play an important role in improving time orientation of children with learning disabilities. When designing interactive multimedia games, designers shall pay attention to not only the clock based time perceptual models, but also the models that do not necessarily require a clock. Technically speaking, all these perceptual models are well 
supported by the computational time models in multimedia systems in one way or the other. It is more a design challenge than a technical one. Three design cases are presented, to show our first attempts in developing interaction, training and diagnostic tools for children with time orientation problems, taking into account the problems in different perceptual time models that relate to the learning disabilities.

Acknowledgements. The authors would like to thank the students who designed the tools and games, among others, Rens Brankaert for retrospective timing, Teun van Roessel for ADHD management in classrooms, and Alice van Beukering, Kyra Frederiks, Wouter van Geesink and Bas van Hoeve for the timer for parent-child interaction.

\section{References}

1. Gibbon, J., Malapani, C., Dale, C.L., Gallistel, C.: Toward a neurobiology of temporal cognition: advances and challenges. Current Opinion in Neurobiology 7, 170-184 (1997)

2. Hazeltine, E., Helmuth, L.L., Ivry, R.B.: Neural mechanisms of timing. Trends in Cognitive Sciences 1, 163-169 (1997)

3. Ivry, R.: Cerebellar timing systems. International Review of Neurobiology 41,555 (1997)

4. Hurksa, P.P.M., Hendriksenbc, J.G.M.: Retrospective and Prospective Time Deficits in Childhood ADHD: The Effects of Task Modality, Duration, and Symptom Dimensions. Child Neuropsychology 17, 34-50 (2010)

5. Pomeroy, S.: 24h Analog Clock Widget (2011, May 1, 2012)

https://play.google.com/store/apps /

details?id=info.staticfree. android. twentyfourhour

6. GoLearn International Inc. TimeBuddy (May 1, 2012), http: / / timebuddy . com

7. BBC. Clockworks, A fun activity to help children at KS1 learn about telling the time (May 1, 2012), http://www.bbc.co.uk/bitesize/ksl/maths/telling_the_time/ play/

8. FreeTrainingTutorial.com. Telling Time Games (May 1, 2012), http: //www. free-training-tutorial.com/telling-time-games.html

9. Grondin, S.: Timing and time perception: a review of recent behavioral and neuroscience findings and theoretical directions. Attention, Perception, \& Psychophysics 72, 561-582 (2010)

10. Zimbardo, P.G., Boyd, J.N.: Putting time in perspective: A valid, reliable individualdifferences metric. Journal of Personality and Social Psychology 77, 1271 (1999)

11. Hu, J.: Design of a Distributed Architecture for Enriching Media Experience in Home Theaters, PhD Thesis, Department of Industrial Design, Eindhoven University of Technology, Eindhoven (2006)

12. Hu, J., Feijs, L.: IPML: Structuring Distributed Multimedia Presentations in Ambient Intelligent Environments. International Journal of Cognitive Informatics \& Natural Intelligence (IJCiNi) 3, 37-60 (2009)

13. Bartneck, C., Hu, J., Salem, B., Cristescu, R., Rauterberg, M.: Applying Virtual and Augmented Reality in Cultural Computing. International Joural of Virtual Reality 7, 11-18 (2008) 